\title{
INFLUENCE OF RESIDUAL STRESSES ON THE FATIGUE LIFE OF A SHOT- PEENED NICKEL-BASED SINGLE CRYSTAL SUPERALLOY: FROM MEASUREMENTS TO MODELING
}

\author{
Amélie Morançais ${ }^{1,3,4}$, Mathieu Fèvre ${ }^{2}$, Manuel François ${ }^{3}$, Nicolas Guel ${ }^{3}$, Serge Kruch ${ }^{1}$, Pascale Kanouté ${ }^{1}$, Arnaud \\ Longuet $^{4}$ \\ ${ }^{1}$ ONERA-The French Aerospace Lab, 29 Avenue de la Division Leclerc, 92322 Châtillon, France \\ ${ }^{2}$ LEM, UMR 104 CNRS-ONERA, 29 Avenue de la Division Leclerc, 92322 Châtillon, Fance \\ ${ }^{3}$ ICD-LASMIS, UMR CNRS 6281 Université de Technologie de Troyes, 12 Rue Marie Curie, 10004 Troyes, France \\ ${ }^{4}$ SAFRAN Snecma Villaroche, Rond-point René Ravaud, 77550 Réau, France
}

Keywords: Residual stress, Single crystal, Superalloy, X-ray diffraction, Lifetime prediction

\begin{abstract}
In this work, X-ray diffraction measurements and finite elements calculations are combined to investigate the effect of the shot-peening process on the fatigue lifetime of the AM1 nickel-based single crystal superalloy. The Ortner method is used to determine residual elastic stress depth profiles in plane-parallel samples. They exhibit a 130-160 $\mu \mathrm{m}$ thick hardened layer where there are compressive stresses up to 1000-1400 MPa. The tensile stresses which ensure the mechanical equilibrium of the samples are not localized in a specific layer but rather distributed in a few millimeters thick layer. The eigenstrain theory is then used to incorporate measured stresses in the elasto-viscoplatic modeling of shot-peened fatigue test specimens. A numerical method is proposed to initialize hardening variables in the shot-peened layer independently of the complexity of the constitutive law or measurements in calibration samples. Finally, a fatigue analysis at $650{ }^{\circ} \mathrm{C}$ is performed in samples with a stress-concentration. The effect of shot-peening on the fatigue lifetime is studied using both modeling and measurements. Results are in good agreement in the investigated range of applied stresses. However, measurements show that residual stresses from shot peening are not always beneficial.
\end{abstract}

\section{Introduction}

The fatigue analysis of blades in the hot stage of aeronautical gas turbines is extensively investigated to improve the engine reliability. Life prediction of these components under complex thermomechanical loading and environmental conditions is nowadays part of the design process undertaken by engine manufacturers. Finite element calculations at the level of the component are able to identify fatigue critical zones and crack initiation time depending on its thermal and mechanical history [1]. The development of a consistent approach connecting the physical variables required to describe the mechanical behavior of the material to those required to describe the fatigue process is still a challenging task because damage is closely connected to the microstructure of the material. For blades made of AM1 nickel-based single crystal superalloy, a lifetime analysis has been developed since the 1980's in the framework of continuum damage mechanics. The elasto-viscoplastic model, which enables the prediction of the anisotropic behavior of the single crystal at different temperatures, is coupled to a creep-fatigue damage model $[2,3,4]$. To delay crack initiation and propagation and thus improve fatigue lifetime, turbine blade roots are shot-peened. The compressive residual stress layer and the plastic deformation created at the surface of the component contribute to relax stress concentration caused by the fir-tree geometry. Due to the complex thermomechanical loading endured by blades during service, one may wonder in which circumstances the shot-peening treatment is really beneficial.

The aim of this study is to take into account stress fields and work hardening generated by shot-peening in the finite element code Zset/Zebulon used by SNECMA (SAFRAN group) and ONERA for modeling cyclic fatigue in the AM1 single-crystal superalloy. The ultimate goal of this work is to get efficient estimations of the benefit of the shot-peening treatment on fatigue lifetime. The paper is organized as follows. The first section is devoted to the determination of elastic stresses using the Ortner method [6]. After a description of the formalism and technical details, stress measurements on specimens with shot-peened flat surfaces oriented along the $<100>$ or the $<110\rangle$ crystallographic directions are presented. In the second section, the procedure used to incorporate stresses in the finite element calculations is described and applied to generate the initial mechanical state of a shot-peened single crystal fatigue test specimen. In the last section, the low cycle fatigue lifetime of specimens exhibiting a stress concentration is calculated 
and compared to measurements.

\section{Residual stress determination in single crystals using X-ray diffraction}

Formalism and methodology

The strain of the crystal lattice is determined by X-ray diffraction measurements. The elastic stress tensor $\boldsymbol{T}$ is then deduced with the generalized Hooke law:

$$
T=C: E
$$

where $\boldsymbol{C}$ and $\boldsymbol{E}$ are the elastic stiffness and strain tensors of the crystal lattice. In the case of single crystals, the the Ortner method can be utilized [6] when a monochromatic wavelength is used. The GreenLagrange strain tensor components $E_{i j}$ are related to the metric tensor components $g_{i j}$ and $g_{i j}^{0}$ of the deformed and undeformed crystal lattices by:

$$
E_{i j}=\frac{1}{2}\left(g_{i j}-g_{i j}^{0}\right)
$$

where $g_{i j}=\mathbf{a}_{i} \cdot \mathbf{a}_{j}$ with $\mathbf{a}_{i}(\mathrm{i}=1,2,3)$ the crystal lattice basis vectors.

Diffraction techniques involve measurements in the dual (reciprocal) space of the crystal lattice. By definition of the reciprocal space basis $\left\{a_{i}^{*}\right\}(\mathrm{i}=1,2,3)$, the components of its metric tensor $g_{i j}^{*}=\mathbf{a}_{i}^{*} \cdot \mathbf{a}_{j}^{*}$ are obtained by inverting the $g_{i j}$ matrix:

$$
\left[g_{i j}^{*}\right]=\left[g_{i j}\right]^{-1}
$$

and are related to diffraction angles by:

$$
\sum_{i, j=1}^{3} h_{i} h_{j} g_{i j}^{*}=d_{h}^{-2}=\frac{4 \sin ^{2} \theta_{h}}{m^{2} \lambda^{2}}
$$

where the coefficients $\left\{h_{i}\right\}$ correspond to the Miller indices of the lattice plane associated with the node of the reciprocal lattice designated by the vector $\mathbf{h}, \theta_{h}$ is the angle between the incident beam and the scattering planes, $m$ is an integer corresponding to the order of the reflection and $\lambda$ is the wavelength of the incident wave. In principle, six Bragg angles corresponding to non colinear $\mathbf{h}$ vectors have to be measured in order to fully determine the metric tensor. However, in order to reduce the effect of uncertainties on the accuracy of the solution, the number of lattice planes $N$ is usually taken larger $[7,8]$. The best solution in the sense of least-square minimization is obtained by solving the normal equations and the six independent coefficients of the reciprocal space metric tensor $g_{i j}^{*}$ are given by:

$$
\left[g^{*}\right]=\left([h]^{T}[h]\right)^{-1}[h]^{T}\left[d^{-2}\right]
$$

with

$$
[h]=\left[\begin{array}{cccccc}
h_{1}^{2} & k_{1}^{2} & l_{1}^{2} & 2 k_{1} l_{1} & 2 h_{1} l_{1} & 2 h_{1} k_{1} \\
h_{2}^{2} & k_{2}^{2} & l_{2}^{2} & 2 k_{2} l_{2} & 2 h_{2} l_{2} & 2 h_{2} k_{2} \\
\vdots & & & & & \vdots \\
h_{N}^{2} & k_{N}^{2} & l_{N}^{2} & 2 k_{N} l_{N} & 2 h_{N} l_{N} & 2 h_{N} k_{N}
\end{array}\right]
$$

$$
\begin{aligned}
{\left[g^{*}\right] } & =\left[g_{11}^{*}, g_{22}^{*}, g_{33}^{*}, g_{23}^{*}, g_{13}^{*}, g_{12}^{*}\right]^{T} \\
{\left[d^{-2}\right] } & =\left[\frac{4 \sin ^{2} \theta_{1}}{m^{2} \lambda^{2}}, \cdots, \frac{4 \sin ^{2} \theta_{N}}{m^{2} \lambda^{2}}\right]^{T}
\end{aligned}
$$

where $h_{i}, k_{i}$ and $l_{i}$ are the Miller indices of the $i$ th lattice plane used for the measurement. To minimize numerical errors, the number of lattice planes and their orientation relationship must be chosen such that the condition number associated with the linear equation 5 is small $[7,8]$.

The methodology applied in the following to determine residual stress depth profiles can be summarized as follows:

- measurement of $N>6$ Bragg angles

- calculation of $g_{i j}$ using equation 5 and equation 3

- calculation of the crystal lattice stress tensor $T_{i j}$ using equation 2 and equation 1

- calculation of the stress tensor in the reference system of the specimen

- material removal by electropolishing and iteration of the procedure.

Material and experimental details

Samples Electro Discharge Machining (EDM) is used to obtain $15 \mathrm{~mm}$ x $6 \mathrm{~mm}$ x $6 \mathrm{~mm}$ plane-parallel samples from a bar of the AM1 nickel-based single crystal produced by directional solidification at SNECMA. For all samples, the $6 \mathrm{~mm} \times 6 \mathrm{~mm}$ surfaces are oriented along the [010] crystallographic direction which corresponds to the solidification axis and the primary arms axis of dendrites. Other surfaces have $<100>$ (Figure 1a) or $\langle 110\rangle$ (Figure 1b) crystallographic orientations depending on the batch of samples. Optical microscopy observations reveal dendrites with a primary arms separation in the order of $500 \mu \mathrm{m}$ (Figures 1c,1d). Porosity due to the solidication process and coarse $\gamma^{\prime}$ particles which have not been entirely removed by the solution heat treatment are also present (black and white spots). Shot-peening and diffraction measurements are realized on one of the $15 \times 6 \mathrm{~mm}^{2}$ surfaces. To remove the re-cast 
(a)

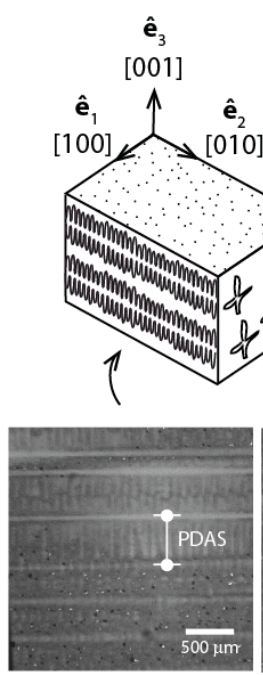

(c) (b)
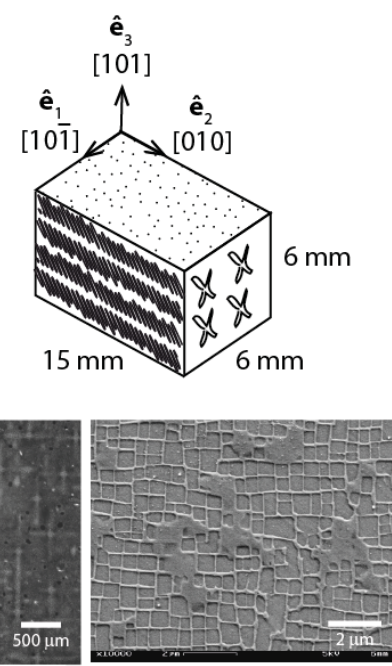

(e)

Figure 1. Schematics of the relationship between sample geometry, dendrites and crystallographic orientations of investigated samples. $\left(\hat{\mathbf{e}}_{1}, \hat{\mathbf{e}}_{2}, \hat{\mathbf{e}}_{3}\right)$ designates the Cartesian coordinate system used to express tensor components. Shot-peening and X-ray diffraction measurements are realized on the dotted surface which is oriented along (a) the [001] crystallographic direction or (b) the [101] crystallographic direction. (c) and (d) are optical micrographs of sample sides where "PDAS" denotes the Primary Dendrite Arm Spacing. Black and white spots represent porosity and eutectic phases respectively. (e) is a Scanning Electron Microscopy micrograph of the cuboidal $\gamma^{\prime}$ precipitates (dark grey) surrounded by the $\gamma$ matrix (light grey).

layer and the residual stresses which may have been introduced by the EDM, this surface is subjected to a soft mechanical polishing. Solution and ageing heat treatments are then applied to obtain an austenitic nickel rich $\gamma$ matrix strengthened by about $70 \%$ volume fraction of $\gamma^{\prime}-\mathrm{Ni}_{3} \mathrm{Al}$ ordered precipitates with a cubic $\mathrm{L}_{2}$ structure. $\gamma^{\prime}$ precipitates have cuboidal shapes with sizes lower than $1 \mu \mathrm{m}$ and are homogeneously distributed in the $\gamma$ matrix (Figure 1e). The lattice mismatch between the two phases is smaller than $0.05 \%$. The last step of samples preparation is the shot-peening which is is realized with $1 \mathrm{~mm}$ diameter 100Cr6 steel shots at $100 \%$ coverage by an ultrasonic technique.

Measurements conditions and data analysis

The diffraction measurements are realized using a diffractometer composed of a Rigaku microfocus X-ray generator equiped with parabolic optics $(\mathrm{Cu}$ wave-

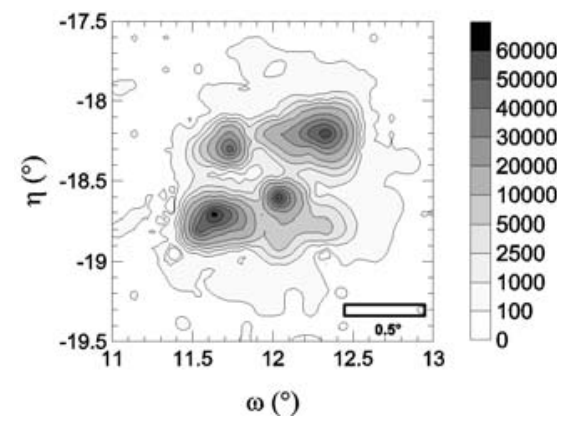

Figure 2. ( $\omega, \eta)$ intensity maps (counts per second) around the $(\overline{1} 33)$ reflexion in the reference sample at at depth of $202 \mu \mathrm{m}$.

lengths), a general purpose Huber 6-circles goniometer and a $\mathrm{NaI}(\mathrm{Tl})$ scintillation detector located at 700 $\mathrm{mm}$ from the sample position. The opening of the divergence and detector crossed slits is set to $1 \mathrm{~mm}$ $\times 1 \mathrm{~mm}$ in order to have a good signal to noise ratio when high angles diffraction peaks are investigated in shot-peened samples. Once the diffraction condition has been found for a given $(\mathrm{hkl})$ reflection, all the goniometer motor positions are recorded and a $\theta-2 \theta$ scan is realized to obtain the diffraction peak. Data are then corrected by the Lorentz-polarisation factor and scans are analyzed with a least-square fitting procedure using a Rachinger doublet [9]. As explained above, the knowledge of the Miller indices and the Bragg angle of at least six reflections enables to determine the metric tensor of the crystal unit cell, thus the six parameters of the Bravais lattice.

Due to the small lattice mismatch between the $\gamma$ and $\gamma^{\prime}$ phases, the contribution of the two phases in (hkl) diffraction peaks with $h, k, l$ having the same parity are not differentiated using our experimental setup. In principle, additional monochromators would probably help but with higher X-ray beam fluxes. Also, the large peak broadening caused by shot-peening would not guarantee a quantitative deconvolution of the two contributions. Therefore, our analysis of fundamental reflections provide the average strain between the $\gamma$ and $\gamma^{\prime}$ phases. The best strain sensitivity and accurate solutions of equation 5 are obtained with reflections diffracting at high Bragg angles. In this work, $\{420\}$, $\{331\},\{004\}$ and $\{222\}$ reflections are considered. This corresponds to diffraction angles close to $147^{\circ}, 138$ ${ }^{\circ}, 118^{\circ}$ and $96^{\circ}$ respectively. 21 Bragg angles are used in the calculations when the sample surface is oriented along the [100] crystallographic direction, 20 when the surface is oriented along the [110] direction. To determine the residual stress tensor components, a macroscopic stiffness tensor determined from me- 


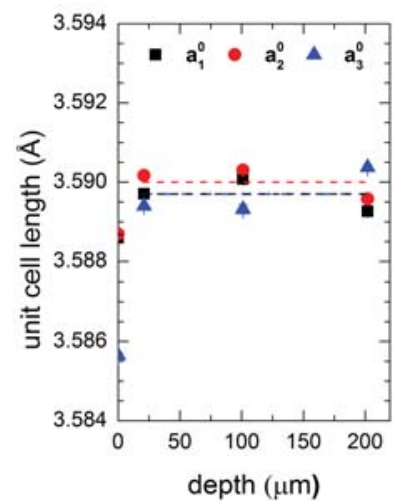

(a)

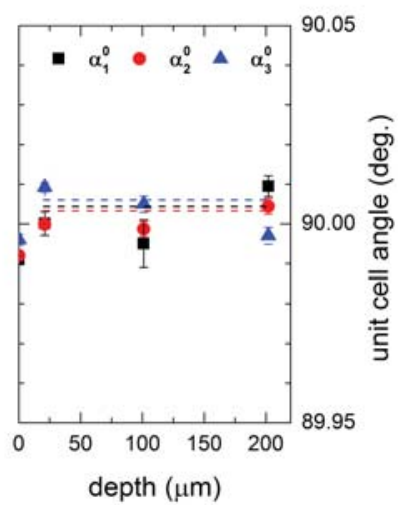

(b)
Figure 3. Unit cell lattice parameters determined in a sample which has not been subjected to shot-peening. (a) cell lengths (b) cell angles $\alpha_{i}=\left(\widehat{\mathbf{a}}_{j, \mathbf{a}}\right)$

chanical testing measurements at room temperature is used: $C_{1111}=296 \mathrm{GPa}, C_{1122}=204 \mathrm{GPa}$ and $C_{2323}=125$ GPa [11]. Superstructure reflections are related to the $\gamma^{\prime}$ phase only and have intensities more than 1000 times smaller than fundamental reflections [12]. For the shotpeened specimen, crystal misorientations near sample surface reduce significantly the maximum intensity of Bragg peaks resulting in intensities comparable to the noise intensity. Thus, our setup is not able to provide a complete residual strain depth profile related to the $\gamma^{\prime}$ phase.

The evaluation of uncertainties is performed through a Monte-Carlo approach, assuming random fluctuation around the measured diffraction angle. Uncertainties include lattice parameter variations due to composition fluctuations, mosaic spread introduced by the dendrites and errors arising from the least-squares fitting procedure of the Bragg peaks. Details are provided in [12].

\section{$\underline{\text { Results and discussion }}$}

Two methods are commonly used to determine the metric tensor $\boldsymbol{g}^{0}$ of the undeformed state: measurements of the unit cell parameters in a reference sample or, for low penetrating radiations, the plane stress approximation, where the lattice parameter of the reference state is taken such that the component $T_{33}$ of the stress tensor related to the deformed state is zero (with $\hat{\mathbf{e}}_{3}$ direction along the surface normal of the sample). For the $\mathrm{Cu}$ wavelength, the $\mathrm{X}$-ray attenuation length in the superalloy is close to $10 \mu \mathrm{m}$. Depending on the orientation of the sample with respect to the incoming and diffracted beams, the characteristic depth probed ranges from $2 \mu \mathrm{m}$ for grazing angles to $10 \mu \mathrm{m}$ close to normal angles. Both methods are assessed in this work.

Marty et al. and Brückner et al. have shown that in the diffraction condition, several local maxima are visible due to the misorientation between dendrites $[13,14]$. This is illustrated in Figure 2. In such case, $\theta-2 \theta$ scans are recorded for $n$ local maxima, with $n \leq 4$ in order to quantify the variations of the lattice plane spacing. The strain is calculated from the middle of the variation range of peak positions, while the error bar is computed from half the range. After an optimization work detailed in [12], we show that the lattice parameter fluctuations related to the dendritic microstructure and the choice of reflections in the calculations result in $30 \mathrm{MPa}$ uncertainties on the calculated stress tensor components.

Reference sample Figure 3 represents the six unit cell parameters $\left(\mathbf{a}_{i}, \alpha_{i}\right)$ as a function of depth in a heat treated reference sample with its surface normal oriented along the $\{100\}$ crystallographic directions. Each unit cell length oscillates as a function of depth with an average of $3.5897 \AA$ or $3.5900 \AA$ (dashed lines) with maximum deviations of $\pm 4 \cdot 10^{-4} \AA$. At the sample surface, lattice constants differ significantly probably due to chemical inhomogeneities and/or remaining stresses caused by the heat treatments and the mechanical polishing respectively. The reference state can be assimilated to a cubic crystal with a lattice parameter $a^{0}=3.58985 \AA$ which leads to:

$$
g_{i j}^{0}=\left(a^{0}\right)^{2} \delta_{i j}=3.58985^{2} \delta_{i j}
$$

where $\delta_{i j}$ is the Kronecker delta.

Sample with a $\{100\}$ shot-peened surface Residual elastic stress depth profiles resulting from sets of measurements and layer removals are represented in Figure 4 using the metric tensor of equation 9 (Figure $4 \mathrm{a}$ ) and the plane stress approximation (Figure $4 \mathrm{~b}$ and Figure $4 \mathrm{c}$ ). The evolution of $T_{11}$ and $T_{22}$ which differ by less than $30 \mathrm{MPa}$, shows compressive stresses taking place in the $150 \mu \mathrm{m}$ thick surface layer and almost zero residual stress deeper. The shear components $\left(T_{i j}\right.$ with $\left.i \neq j\right)$ are close to zero for all depths. Although each measurement is performed on a free surface, in the layer affected by the shot-peening process, $T_{33}$ significantly differs from zero when the metric tensor of the reference sample is used (Figure $4 \mathrm{a}$ ). This is probably due to the modification of the material properties (solid solution gradients, loss of coherency strains) in this layer because of the strong hardening caused by shot-peening and the lattice parameter of the reference state should not be taken 

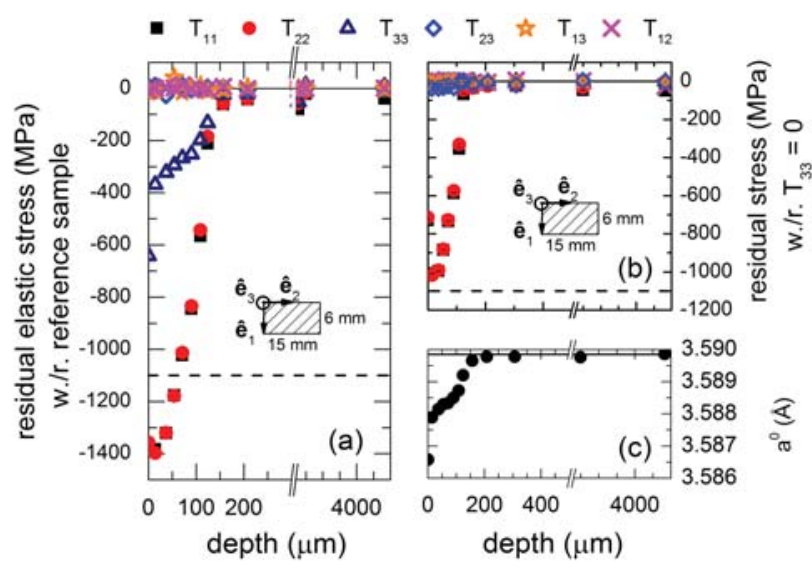

Figure 4. Independent components of the residual stress tensor calculated from measured crystal lattice parameters $\mathbf{a}_{i}$ and $\alpha_{i}(\mathrm{i}=1,2,3)$ as function of depth in the shot-peened sample whose surface is oriented along a $\{100\}$ crystallographic direction. The Cartesian basis is oriented such as $\hat{\mathbf{e}}_{2}$ and $\hat{\mathbf{e}}_{3}$ are colinear with the surface normal and the long edge of the sample (schematics). Dashed line corresponds to the ultimate stress of the AM1 superalloy determined from tensile or fatigue tests at room temperature [15].

like the measured one in the reference sample. In Figure 4, the dashed lines represent the ultimate stress limit of the AM1 material [15]. In Figure 4a stresses close to the sample surface are larger than this value. The maximum difference with this limit is about 300 $\mathrm{MPa}$ at the $20 \mu \mathrm{m}$ depth. This is not incompatible since the material is strongly hardened in this area and if all the tensor components are considered, the von Mises stress is only $60 \mathrm{MPa}$ above the ultimate stress limit. Figure $4 \mathrm{~b}$ represents the residual stress profile determined from the same measurement but with the plane stress approximation $\left(T_{33}=0\right)$. The evolution of the lattice parameter of the reference state (cubic symmetry) as a function of depth is represented in Figure 4c. Up to $200 \mu \mathrm{m}$, its value differs from the lattice parameter of the reference sample (dashed line). As in Figure $4 \mathrm{~b}, T_{11}$ ans $T_{22}$ components are almost equal and shear components close to zero. The highest absolute value is about $1000 \mathrm{MPa}$ close to the surface.

Two additional corrections must be done to the stress profile calculated from Bragg peak positions. The first one to account for the stress relaxations resulting from material removals and the second to account for the fact that the X-ray beam probes a stress gradient. In the latter case, it can be shown that the measured value of the $T_{33}$ component close to the surface plane is twice its true value providing that the stress gradient at the

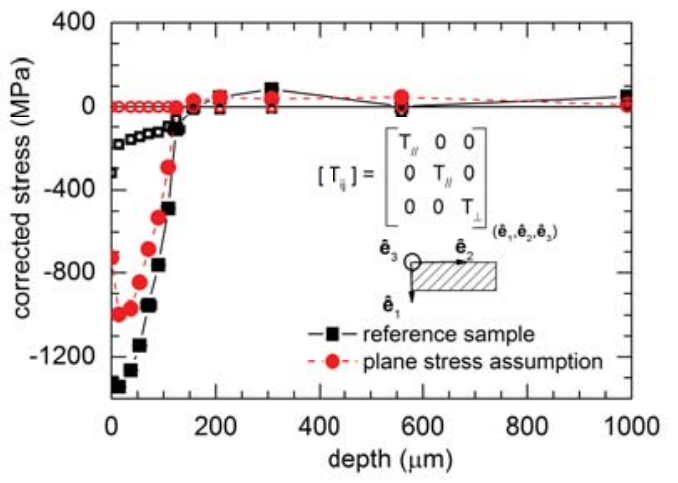

Figure 5. Evolution of residual stresses as a function of depth. Filled symbols correspond to $T_{11}=T_{22}=$ $T_{\|}$and open symbols to $T_{33}=T_{\perp}$. The profile with square symbols is determined with respect to the lattice parameter of a reference sample and circle with respect to the plane stress hypothesis.

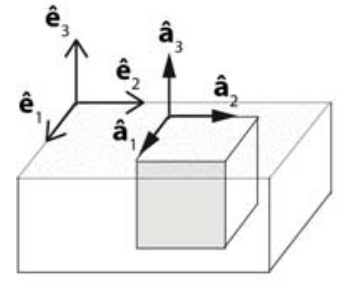

(a)

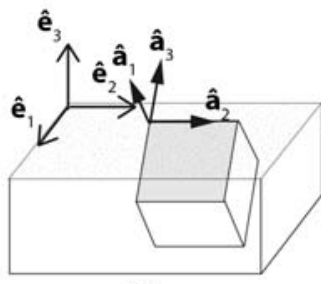

(b)
Figure 6. Relationship between the sample surface and the crystal unit cell orientations when the surface normal of the sample is aligned with (a) the [001] crystallographic direction and (b) with the [101] direction. $\left(\hat{\mathbf{e}}_{1}, \hat{\mathbf{e}}_{2}, \hat{\mathbf{e}}_{3}\right)$ and $\left(\hat{\mathbf{a}}_{1}, \hat{\mathbf{a}}_{2}, \hat{\mathbf{a}}_{3}\right)$ are the sample and crystal Cartesian coordinate systems respectively.

surface is zero (see discussion in [12]). The Moore and Evans correction for stress relaxations due to material removals is applied [16]. The resulting stress profiles are represented in Figure 5. The residual stress depth profile exhibit a 130-160 $\mu \mathrm{m}$ thick hardened layer where $T_{11}$ and $T_{22}$ compressive stresses up to $1000-1400 \mathrm{MPa}$ take place depending on the assumption used to describe the initial state. The tensile stresses which ensure the mechanical equilibrium of the sample are not localized in a specific layer with high levels of stresses but rather distributed in a few millimeters thick layer.

Effects of the crystalline anisotropy To quantify the effect of the crystalline anisotropy on the stress field caused by the shot-peening process, samples with surfaces oriented along the $<100>$ and $<110>$ crystallographic directions have been shot-peened in the same 
Table I. Diagonal components of the $\boldsymbol{T}$ stress tensor at a $20 \mu \mathrm{m}$ depth when the shot-peened sample surface is oriented along the [001] or the [101] crystallographic direction. Values are given in $\mathrm{MPa}$ in the $\left(\hat{\mathbf{e}}_{1}, \hat{\mathbf{e}}_{2}, \hat{\mathbf{e}}_{3}\right)$ sample basis (see Figure 6). The reference state corresponds to a cubic lattice with $a^{0}=3.58985 \AA$ or to the plane stress hypothesis.

\begin{tabular}{|ccccc|}
\hline $\begin{array}{c}\text { Surface } \\
\text { orientation }\end{array}$ & $\begin{array}{c}\text { Reference } \\
\text { state }\end{array}$ & $\mathrm{T}_{11}$ & $\mathrm{~T}_{22}$ & $\mathrm{~T}_{33}$ \\
\hline \hline$[001]$ & reference & -1363 & -1363 & -177 \\
{$[101]$} & sample & -1500 & -1180 & -112 \\
\hline \hline$[001]$ & plane stress & -1010 & -1010 & 0 \\
{$[101]$} & hypothesis & -1275 & -957 & 0 \\
\hline
\end{tabular}

conditions. The relationship between the sample surface and the crystal unit cell orientations is represented in Figure 6. For both orientations, shear components of stress tensors are almost zero (within the $\pm 30 \mathrm{MPa}$ uncertainty) with respect to the $\left(\hat{\mathbf{e}}_{1}, \hat{\mathbf{e}}_{2}, \hat{\mathbf{e}}_{3}\right)$ sample coordinate system. Diagonal components are given in Table. I at a $20 \mu \mathrm{m}$ depth. Within the plane stress hypothesis, if $T_{11}=T_{22}$ for the [100] surface orientation, the two components differ by $28 \%$ for the [101] orientation due to the crystallographic inequivalence between $\hat{\mathbf{e}}_{1}$ and $\hat{\mathbf{e}}_{2}$ directions (see Figure 6). Results also show that the $T_{11}$ component is $265 \mathrm{MPa}$ higher when the surface is oriented along the [101] direction whereas the $T_{22}$ component is $53 \mathrm{MPa}$ lower. When the lattice parameter of the reference sample is used in the calculations, $T_{11}$ and $T_{22}$ differ by $10 \%$ and $14 \%$ between the two sample orientations.

\section{Finite element calculations incorporating residual stresses resulting from shot-peening}

The initial state introduced in the calculations must be as close as possible to the state generated by the shot-peening process and corresponds to a mechanical equilibrium. To reproduce stress redistributions during cyclic loading conditions, internal variables such as eigenstrain, kinematic hardening and accumulated plastic deformation must be carefully initialized in addition to the choice of their governing equations. In the case of single crystals, data obtained from measurements are not able to provide such values for every Gauss point. Therefore assumptions and interpolation schemes are required. In the following, we present a generic approach to initialize internal variables in the layer affected by the shot-peening in the case of single crystals. The method is then applied to investigate the lifetime of a fatigue test specimen made of the AM1 superalloy.

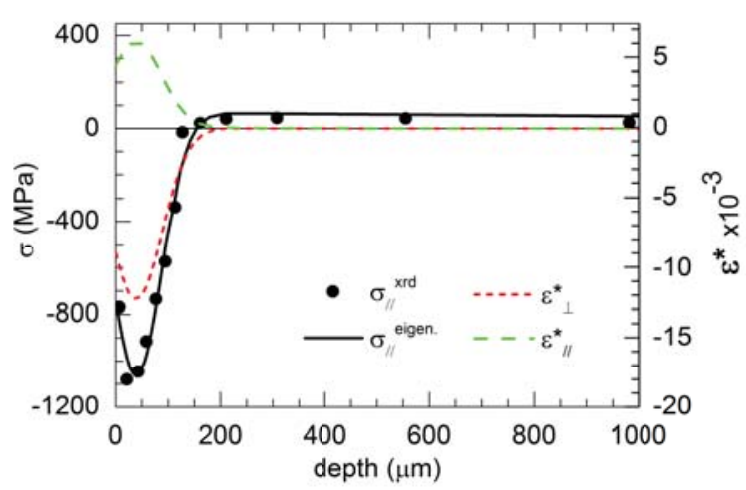

Figure 7. Evolution of residual elastic stresses (left axis) and eigenstrain (right axis) as a function of depth. Circles corresponds to X-ray diffraction measurements and lines to modeling. $X_{\|}$and $X_{\perp}$ denotes in-plane and normal components of $\boldsymbol{X}$ tensor.

\section{Internal variables initialization}

Eigenstrain. The eigenstrain framework enable to account for strains caused by a wide range of phenomena such as thermal expansion mismatch, phase transformation or plastic deformation. The formalism can be easily implemented in finite element calculations and a detailed modeling of the process generating residual stresses is not required when measurements are available. The method is extensively used to investigate stresses in shot-peened components mainly for the following reasons: (1) for a given eigenstrain distribution, an equilibrated residual stress distribution can be calculated for any geometry, (2) the residual stress state evolution during an additional plastic activity in the material is able to be modeled through the modification of the eigenstrain distribution, (3) the eigenstrain distribution related to the shot-peening process can be determined from residual stress measurements on samples with simple geometries and then be incorporated into the modeling of components with more complex geometries.

In the small strain approximation, the mechanical equilibrium of a system with stress free boundary conditions at the surfaces is obtained by solving the following set of equations:

$$
\left\{\begin{array}{l}
\text { Inc } \boldsymbol{\epsilon}=0 \\
\nabla \cdot \boldsymbol{\sigma}=0 \\
\boldsymbol{\sigma} \cdot \mathbf{n}=0 \\
\boldsymbol{\epsilon}=\boldsymbol{S}: \boldsymbol{\sigma}+\boldsymbol{\epsilon}^{*}
\end{array}\right.
$$

where $\boldsymbol{\sigma}$ is the residual stress tensor, $\boldsymbol{\epsilon}$ is the total strain tensor, $\boldsymbol{S}=\boldsymbol{C}^{-1}$ is the compliance tensor, $\boldsymbol{\epsilon}^{*}$ is the eigentrain tensor and $\mathbf{n}$ correponds to the surface normal. The shot peening being operated in normal conditions, 
we assume that only the stress tensor components in the surface plane are non zero. The solution of equations 10 for a plate-like sample which has been shot-peened on a face oriented along the $\hat{\mathbf{e}}_{3}$ direction has the following expression:

$$
\left\{\begin{array}{l}
\epsilon_{11}^{*}(z)=A_{1} z+B_{1}-\left[S_{11} \sigma_{11}(z)+S_{12} \sigma_{22}(z)\right] \\
\epsilon_{22}^{*}(z)=A_{2} z+B_{2}-\left[S_{12} \sigma_{11}(z)+S_{22} \sigma_{22}(z)\right] \\
\epsilon_{33}^{*}(z)=-\left[\epsilon_{11}^{*}(z)+\epsilon_{22}^{*}(z)\right]
\end{array}\right.
$$

with

$$
\begin{aligned}
A_{i} & =\frac{12}{h} \int_{-h / 2}^{h / 2} z \epsilon_{i i}^{*}(z) d z \\
B_{i} & =\frac{1}{h} \int_{-h / 2}^{h / 2} \epsilon_{i i}^{*}(z) d z=<\epsilon_{i i}^{*}>_{z}
\end{aligned}
$$

where $h$ is the sample thickness. As illustrated in Figure 7 , the residual elastic stress depth profile of Figure 5 is easily modeled if a Gaussian function is assumed for the $z$ dependence of $\epsilon_{11}^{*}(z)$ and $\epsilon_{22}^{*}(z)$ (dashed lines in the figure).

Kinematic hardening and accumulated plasticity. The method is inspired by the work of Grasty and Andrew [17] where the upper layers of the system are subjected to a squeeze pressure such that a small plastic deformation is generated. This process was iterated by the authors until a known curvature caused by the indentation process is retrieved. Here, we use a similar strategy in the sense that, at different depths, a pressure value is looked for in such a way that after release eigenstrain values determined previously are obtained. The pressure is applied using a serrated function of time until the mechanical state has reached the equilibrium and the accumulated plastic deformation a target value, which is material history dependent. Here, as a rough approximation, diffraction peak widths recorded in shot-peened samples are compared to peak widths evolution determined in calibration samples with a known amount of macroscopic plastic deformation. To reduce calculation costs, this procedure is applied only to a finite set of representative volume elements (RVE) chosen at different locations in the system of interest. The values of variables resulting from independent calculations are then associated with the considered Gauss point.

Crystalline anisotropy. To take into account the effect of the crystalline anisotropy, the procedure developed above can be applied to residual elastic stress measurements in samples with different surface orientations. However, if data are not available, the equivalent pressure during the peening operation can be supposed independent of the crystallographic orientation of the sample surface. This implies that the pressure value and

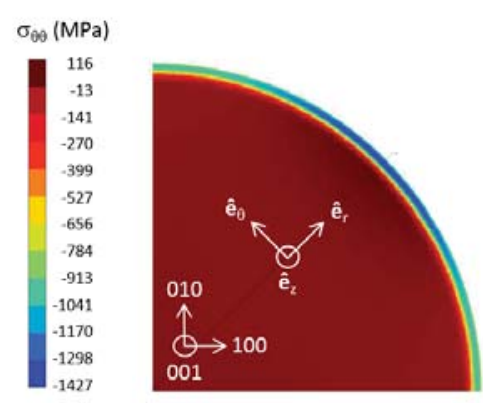

(a)

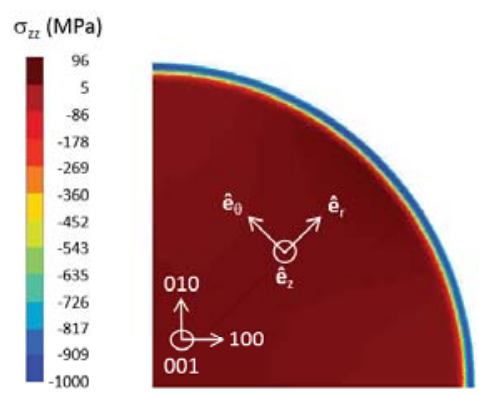

(c)

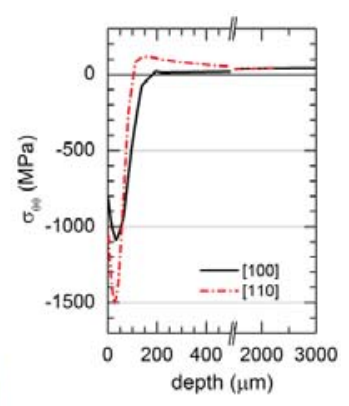

(b)

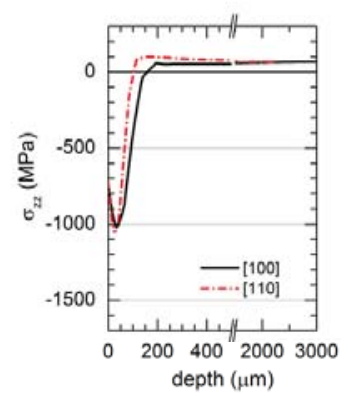

(d)
Figure 8. Calculated hoop (a) and axial (c) residual stress maps in a shot-peened cylindrical fatigue test specimen made of the AM1 single-crystal superalloy at room temperature. Only a quarter of cross-section is shown. (b) and (d) graphs correspond to the stress depth profiles along the [100] and [110] crystallographic directions.

the number of cycles determined for the [100] crystallographic direction can be used to determine eigenstrain, kinematic hardening, accumulated plastic deformation and residual elastic stress values for all crystal orientations. This methodology is applied to a fatigue test specimen with a cylindrical geometry (3.11 mm radius). Room temperature behavior has been calculated using the anisotropic elasto-viscoplatic model developed for the AM1 superalloy [18]. Figure 8 represents the hoop $\left(\sigma_{\theta \theta}\right)$ and axial $\left(\sigma_{z z}\right)$ stresses in a quarter of crosssection just after shot-peening. The hoop stress is significantly affected by the crystalline anisotropy since in Figure 8a, stresses differ by about $500 \mathrm{MPa}$ between the [100] and [110] crystal directions in the shot-peened layer. In the case of the [100] direction, the depth profile exhibit compressive stresses up to $1000 \mathrm{MPa}$ in a $150 \mu \mathrm{m}$ thick layer and tensile stresses are smoothly distributed with depth (solid line in Figure 8b). In the case of the [110] direction, compressive stresses up to $1500 \mathrm{MPa}$ take place in a $100 \mu \mathrm{m}$ thick layer and a tensile stress layer is clearly visible. The axial stress behavior is less affected by the crystalline anisotropy as 
shown in Figure 8c and Figure 8d (dashed line). Calculations only show that the layer where compressive stresses take place is thinner in the [110] direction than in the [100] direction. At a $20 \mu \mathrm{m}$ depth, the calculated hoop component is $34 \%$ lower in the [110] direction than in the [100] direction. This value has to be compared with $28 \%$ in the case of measurements in flat samples (see Table I). The axial stress is $10 \%$ lower in the calculations whereas it is $5 \%$ higher in experiments. X-ray measurements in a shot-peened fatigue specimen are in progress to assess the relevance of trends observed in calculations.

\section{Towards fatigue lifetime assessment}

The ultimate goal of this work is to get efficient estimations of the benefit of the shot-peening treatment on fatigue lifetime. The generated residual stresses that influence the mechanical state at the surface of the part may evolve with the development of the plasticity in the component during cyclic loading conditions and work hardening will also play a significant role on this stress relaxation. Indeed, in experiments, a relaxation of the residual stresses close to $200 \mathrm{MPa}$ is observed after an isothermal ageing at $650{ }^{\circ} \mathrm{C}$ and a relaxation close to $300 \mathrm{MPa}$ after a fatigue loading applied until failure at the same temperature. The model, as it is identified, reproduces correctly the mechanical relaxation but underestimates the thermal one. Parameters of the recovery term acting on the back stress need to be adapted to improve the predictions of the model. Residual stresses and work hardening need then both to be taken into account in the fatigue analysis. The method proposed in the previous section to introduce theses quantities in a finite element mechanical analysis in fatigue allows for an assessment of their combined and separated contribution. In the following, the method is used to estimate the low cycle fatigue lifetime to crack initiation at $650^{\circ} \mathrm{C}$ on notched specimens made of the AM1 single crystal superalloy.

The fatigue life analysis of structures more often is based on the use of a damage model to describe the initiation and coalescence of micro-cracks leading to an observable macroscopic crack. The damage evolution usually expressed in terms of damage increment per cycle is then introduced into a lifetime workflow. As admitted generally for metallic materials, this workflow can be built assuming an uncoupled formalism between the descriptions of the viscoplasticity and hardening mechanisms occurring in the materials and the damage evolution [19]. However, interaction between damage mechanisms induced by fatigue and creep is often considered eventually coupled with oxidation effects [3]. The life-
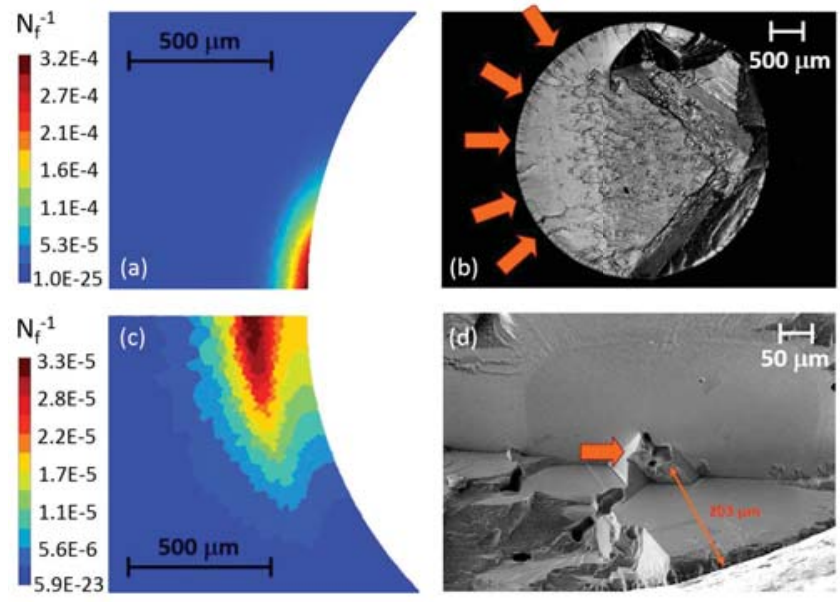

Figure 9. Left - local damage maps calculated model in notched cylindrical fatigue test samples with a stressconcentration factor $K_{t}=1.6$ subjected to an applied stress of $800 \mathrm{MPa}$ at $650{ }^{\circ} \mathrm{C}\left(\mathrm{f}=15 \mathrm{~Hz}, \mathrm{R}_{\sigma}=0\right)$. Zoom in the crack initiation area in (a) a smooth sample (c) a shot-peened sample. Views are mirrored for sake of comparison. Right - fracture SEM micrographs in corresponding fatigue test specimens.

time assessment of the fatigue samples considered in this study is then performed following several steps briefly presented hereafter. Firstly, the procedure described before is used to introduce in the finite element model the initial profiles of the residual stresses and all the hardening variables considered in the constitutive model, chosen to simulate the mechanical behavior of the material. Then, the fatigue loading sustained by the specimens presenting a stress concentration $\left(\mathrm{K}_{t}=1.6\right.$ in the gauge length) is simulated to account for the stress redistributions due to cyclic plasticity namely in the affected layer by the shot-peening process. At this stage it is particularly important that the constitutive model chosen to describe the behavior of the material allows for an accurate simulation of both the residual stress relaxation and the work hardening evolution. Determination of their profile after interrupted fatigue tests may be necessary and a specific formulation of the kinematic hardening variables describing Bauschinger effects can be used [4]. Finally, the damage model is applied as a post-processing on mechanical quantities obtained at the stabilized cycle such as for instance the octahedral shear stress amplitude, the mean value of the hydrostatic stress and the maximum stress eigen to compute the fatigue life and to get the crack localization in the structure. For the analysis performed in this study, a multiaxial creep-fatigue damage model has been used to compare the estimated lifetime between smooth and shot-peened specimens [20]. The fatigue damage model 


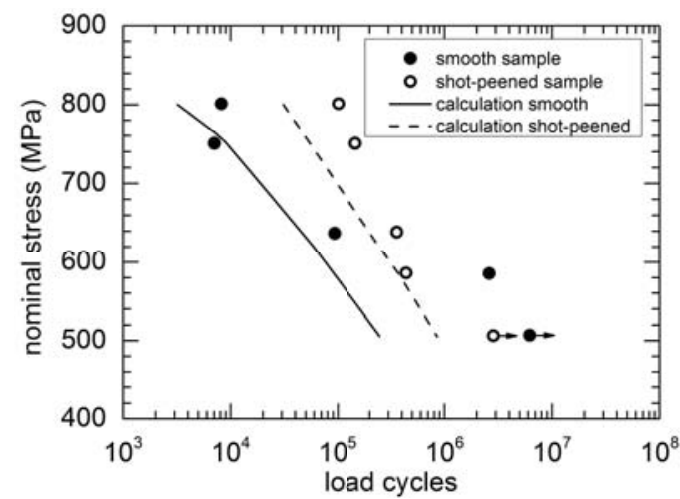

Figure 10. $\mathrm{S} / \mathrm{N}$ curve of $\mathrm{K}_{t}=1.6$ fatigue test specimen made of the AM1 single crystal superalloy $\left(\mathrm{T}=650{ }^{\circ} \mathrm{C}\right.$, $\mathrm{f}=15 \mathrm{~Hz}, \mathrm{R}_{\sigma}=0$ ). Circles corresponds to measurements and lines to modeling. Arrows indicate measurements stopped before failure.

is stress based, involving the Von Mises invariant to describe the maximum stress amplitude and the hydrostatic stress to include the influence of the mean stress on the fatigue predictions. The creep damage model is based on the formalism proposed by Hayhurst [21] which is also a combination of stress invariants. A zoom of the local damage map is represented in Figure 9 for both type of samples. Calculations correspond to a cyclic fatigue test at $650{ }^{\circ} \mathrm{C}$ and a $800 \mathrm{MPa}$ applied stress. In the case of the smooth specimen, the maximum of damage is localized at the sample surface and corresponds to 3000 cycles before crack initiation (Figure 9a). The computed number of cycles for the crack initiation corresponds to the maximum value of the damage parameter, generally set to 1 . The effect of shot-peening is clearly visible in Figures $9 \mathrm{c}$ since the maximum of damage is localized about $200 \mu \mathrm{m}$ beneath the surface and corresponds to a number of cycles one order of magnitude higher. These trends are corroborated by the scanning electron micrographs which show that cracks initiate at the sample surface for the smooth specimen and at a $200 \mu \mathrm{m}$ depth for the shot-peened specimen (arrows in Figure 9b and Figure 9d). Numerical and experimental results obtained for different levels of nominal applied stress are represented in Figure 10. Calculated and experimental lifetimes are in good agreement when the shot-peening process has beneficial effects. The lifetime is 10 times higher at $800 \mathrm{MPa}$ and 4 times higher at $635 \mathrm{MPa}$. However, in measurements a crossover is observed between $585 \mathrm{MPa}$ and $635 \mathrm{MPa}$ since the lifetime is lower for shot-peened samples. This tends to show that the sample surface roughness may be the limiting factor for the fatigue lifetime in the small applied stress regime.

\section{Conclusion}

In this paper, X-ray diffraction measurements and finite element calculations are performed to study the effect of shot-peening on the low cycle fatigue of a nickel-based single crystal superalloy (AM1). In the modeling, the mechanical state associated with the shot-peened operation is incorporated using the results of the residual elastic stress measurements, the eigenstrain framework and a generic procedure which enables to initialize the kinematic hardening and accumulated plastic deformation variables independently of the constitutive equation complexity. A specific approach is also implemented to take into account crystalline anisotropy effects when residual stress depth profiles are not available for all surface orientations. These developments are applied to the lifetime prediction of notched fatigue test samples with a stress-concentration factor $K_{t}=1.6$.

The residual stress depth profile is determined using the Ortner method in a plane-parallel sample with a shot-peened surface oriented along the [100] crystallographic direction. The profile exhibits a $160 \mu \mathrm{m}$-thick hardened layer, where the compressive in-plane stresses are up to 1000-1400 MPa. The tensile stresses which ensure the mechanical equilibrium are smoothly distributed in the sample thickness. Measurements that have been taken in a shot-peened sample with a surface oriented along the [110] crystallographic direction reveal a $30 \%$ increase of compressive stresses for the component which is not crystallographically equivalent with respect to the [100] surface orientation. The other inplane component of the stress tensor is weakly affected by the crystalline anisotropy. The eigenstrain distribution associated with the residual (elastic) stress profile is then calculated and elastoplastic calculations are performed to initialize the internal variables of the model in the surface layer affected by the shot-peening process. With this approach, a physically justified equilibrated mechanical initial state is generated and the study of stress redistributions during cyclic thermal and mechanical loadings is possible in samples with specific geometries. Finally, the ability of the proposed approach to account for the influence of residual stresses and work hardening on lifetime prediction is assessed in the case of fatigue tests at $650{ }^{\circ} \mathrm{C}$ on samples having a stressconcentration $\left(\mathrm{K}_{t}=1.6\right)$. The results of modeling are in good agreement with measurements in the $635-800 \mathrm{MPa}$ range of applied stress. The increase of the fatigue lifetime due to shot-peening is between a factor 4 and 10 . For smaller applied stress, a deleterious effect of shotpeening is measured probably due to a higher sensitivity of the damage to the surface roughness of samples. 


\section{References}

[1] M. Géradin, J.C. Golinval, and J.P. Mascarell, "Three dimensional turbine blade analysis in thermo-viscoplasticity," Recherche Aérospatiale, 5 (10) (1989), 51-57.

[2] J.-L. Chaboche, and O. Jung, "Application of a Kinematic Hardening Viscoplasticity Model with Thresholds to the Residual Stress Relaxation," Int. J. Plast., 13 (10) (1997), 785-807.

[3] F. Gallerneau, and J.-L. Chaboche, "Fatigue Life Prediction of Single Crystals for Turbine Blade Applications," Int. J. Damage Mech., 8 (4) (1999), 404427.

[4] J.-L. Chaboche, P. Kanouté, and F. Azzouz, "Cyclic Inelastic Constitutive Equations and their Impact on the Fatigue Life Predictions," Int. J. Plast., 35 (2012), 44-66, 2012.

[5] A.M. Korsunsky, "On the modeling of residual stresses due to surface peening using eigenstrain distributions," J. Strain Analysis, 40 (8) (2005), 817824.

[6] Balder Ortner, "Röntgenographische Spannungsmessung an einkristallinen Proben," ed. E. Macherauch and V. Hauk, (Deutsche Gesellschaft für Metallkunde, Oberursel, 1983), 49-68.

[7] B. Ortner, "The choice of lattice planes in X-ray strain measurements of single crystals," Adv. X-ray Anal., 29 (1986), 113-118.

[8] M. François, "Analyse des contraintes résiduelles dans les monocristaux et les matériaux gros grains par diffraction des rayons X" (Master's thesis, ENSCP-Univ. Paris IV, 1987).

[9] W. A. Rachinger, "A Correction for the $\alpha 1 \alpha 2$ Doublet in the Measurement of Widths of X-ray Diffraction Lines," J. Sci. Instrum., 25 (1948), 254-259.

[10] W.R. Busing, and H.A. Levy, "Angle Calculations for 3- and 4- Circle X-ray and Neutron Diffractometers," Acta Cryst., 22 (1967), 457-464.

[11] L. Espié, "Étude expérimentale et modélisation numérique du comportement de monocristaux de superalliages" (Ph.D. thesis, ENSMP, Paris, 1996).

[12] A. Morançais, M. Fèvre, M. François, N. Guel, S. Kruch P. Kanouté, and A. Longuet, "Residual stress determination in a shot-peened nickel-based singlecrystal superalloy using X-ray diffraction," J. Appl. Cryst., 48 (2015), 1761-1776.
[13] B. Marty, P. Moretto, P. Gergaud, J. Lebrun, K. Ostolaza, and V. Ji, "X-ray study on single crystal superalloy SRR99: Mismatch $\gamma / \gamma$ ', mosaicity and internal stress," Acta Materialia, 45 (2) (1997), 791800 .

[14] U. Brückner, A. Epishin, A., T. Link, and K. Dressel, "The influence of the dendritic structure on the $\gamma / \gamma^{\prime}$-lattice misfit in the single-crystal nickel-base superalloy CMSX-4," Mater. Sci. Eng. A, 247 (12) (1998), 23-31.

[15] P. Caron, F. Diologent, and S. Drawin, "Influence of Chemistry on the Tensile Yield Strength of Nickel-Based Single Crystal Superalloys," Adv. Mater. Res., 278 (2011), 345-350.

[16] M.G. Moore, and W.P. Evans, "Mathematical Correction for Stress in Removed Layers in X-ray Diffraction Residual Stress Analysis," SAE Trans., 66 (1958), 340-345.

[17] L.V. Grasty, and C. Andrew, "Shot peen forming sheet metal: finite element prediction of deformed shape," Proceedings of the Institution of Mechanical Engineers, Part B: J. Eng. Manuf., 210 (4) (1996), 361-366.

[18] F. Fournier Dit Chabert, A. Gaubert, A. Longuet and S.Quilici, "Calibration and validation of a constitutive model for single crystal nickel based superalloys" (Paper presented at ODAS 2013, Palaiseau, France, 27-29 May 2013).

[19] S. Kruch, P. Kanouté and V. Bonnand, "ONERAs Multiaxial and Anisothermal Lifetime Assessment for Engine Components," Aerospace Lab Journal, 9 (8) (2015) 1-12.

[20] M. Chaudonneret, "A Simple and Efficient Multiaxial Fatigue Damage Model for Engineering Applications of Macro-Crack Initiation," J. Eng. Mater. Technol., 115 (1993) 373-379.

[21] D. Hayhurst, "Computational Continuum Damage Mechanics: Its Use in the Prediction of Creep in Structures Past, Present and Future," Proceedings of the 5th IUTAM Symposium on Creep in Structures, ed. S. Murakami and N. Ohno (Nagoya, Japan: Kluwer Academic Press 2001), 175-188. 